\title{
A General Framework and Algorithms for Score Level Indexing and Fusion in Biometric Identification*
}

\author{
Takao MURAKAMI ${ }^{\dagger, \dagger \dagger a)}$, Kenta TAKAHASHI ${ }^{\dagger}$, and Kanta MATSUURA $^{\dagger \dagger}$, Members $^{\circ}$
}

\begin{abstract}
SUMMARY Biometric identification has recently attracted attention because of its convenience: it does not require a user ID nor a smart card. However, both the identification error rate and response time increase as the number of enrollees increases. In this paper, we combine a score level fusion scheme and a metric space indexing scheme to improve the accuracy and response time in biometric identification, using only scores as information sources. We firstly propose a score level indexing and fusion framework which can be constructed from the following three schemes: (I) a pseudo-score based indexing scheme, (II) a multi-biometric search scheme, and (III) a score level fusion scheme which handles missing scores. A multi-biometric search scheme can be newly obtained by applying a pseudo-score based indexing scheme to multi-biometric identification. We secondly propose the NBS (Naive Bayes search) scheme as a multi-biometric search scheme and discuss its optimality with respect to the retrieval error rate. We evaluated our proposal using the datasets of multiple fingerprints and face scores from multiple matchers. The results showed that our proposal significantly improved the accuracy of the unimodal biometrics while reducing the average number of score computations in both the datasets.
\end{abstract}

key words: biometric identification, score level fusion, missing score, metric space indexing, pseudo-score, multi-biometric search

\section{Introduction}

Biometric authentication systems, which recognize an individual based on physiological or behavioral characteristics, have been used for various kinds of forensic, government, and commercial applications. There are two different ways to recognize an individual: verification and identification [2]. In verification, a person (referred to as a claimant) claims an identity along with his/her biometric sample (referred to as a query sample). Then, the system computes a score (similarity or distance) between the sample and a biometric template corresponding to the claimed identity (i.e. one-to-one matching) to make a decision whether the claimant is genuine or not. In identification, a claimant only inputs a query sample, and the system computes scores between the sample and templates in the database (i.e. one-tomany matching) to make a decision who the claimant is.

Although biometric identification has long been used

Manuscript received January 16, 2013.

Manuscript revised September 17, 2013.

${ }^{\dagger}$ The authors are with Yokohama Research Laboratory, Hitachi, Ltd., Yokohama-shi, 244-0817 Japan.

${ }^{\dagger \dagger}$ The authors are with Institute of Industrial Science, The University of Tokyo, Tokyo, 153-8505 Japan.

${ }^{*}$ A preliminary version of this paper was presented at the International Joint Conference on Biometrics (IJCB 2011), Washington DC, USA, October, 2011 [1].

a)E-mail: takao.murakami.nr@hitachi.com

DOI: 10.1587/transinf.E97.D.510 for criminal investigations, nowadays it is also used for commercial applications (e.g. computer login or physical access control) because of its convenience: it does not require a claimant to input an ID number nor to present a smart card. Above all, remote biometric systems (e.g. face or iris recognition at a distance) [3], which identify a claimant at a distance, can provide the most convenient way of authentication among all the authentication systems because the claimant is not required to perform any action to be recognized.

However, in biometric identification, the following two problems grow bigger as the number of enrollees $N$ increases: (1) the identification error rate increases because the number of candidates increases; (2) the one-to-many matching time also increases because the number of score computations increases. These problems make biometric identification quite challenging to apply to the large-scale applications.

There are several approaches to improving the accuracy or response time in identification (as described in detail in Sect. 2.2). Among them, a score level fusion scheme [4] and a metric space indexing scheme [5], [6] only use scores as information sources to improve the accuracy and the response time, respectively. A score level fusion scheme combines scores obtained from multiple modalities (e.g. fingerprint, face, and iris; index and middle fingerprints; multiple matchers). On the other hand, a metric space indexing scheme reduces the number of score computations using scores as a clue. The main feature of them is general applicability: since both of them only use scores as information sources, they can be applied to any kind of biometric system that outputs scores. Although a number of score level fusion schemes [4] and metric space indexing schemes [5], [6] have been proposed so far, no studies have ever tried to combine the two schemes, to solve the above two problems at the same time, to the best of our knowledge.

To aim of this paper is to develop a generally applicable method to improve the accuracy and response time in biometric identification. To achieve this aim, we make the first-ever attempt (to the best of our knowledge) to combine a score level fusion scheme and a metric space indexing scheme. The main contributions of our work are as follows:

- We propose a score level indexing and fusion framework which can be constructed from the following three schemes:

(I) a metric space indexing scheme which computes 
score-like values (referred to as pseudo-scores), which can be quickly computed and are relevant to scores, between a query sample and templates [7]-[10]. We refer to such a scheme as a pseudo-score based indexing scheme;

(II) a multi-biometric search scheme which selects some templates from the database, using pseudoscores and scores as a clue, and computes scores for them.

(III) a score level fusion scheme which handles missing scores [11]-[16].

This framework clarifies the generality of our approach: we can use any existing scheme as (I) or (III). At the same time, it also clarifies the novelty of our work: (II) is a new scheme which is necessary to construct our framework.

- We propose the NBS (Naive Bayes search) scheme as (II). The experimental results showed that our proposal using the NBS scheme significantly improved the accuracy of the unimodal biometrics, while reducing the number of score computations.

This paper is an extension of a previously published conference paper[1]. The main enhancements are as follows:

- This paper clarifies the optimal property of the NBS scheme with respect to search efficiency (Lemma 1), and discusses it (Sect. 4.2.2).

- This paper proposes a technique for reducing the average number of score computations without significantly affecting the accuracy, by identifying the claimant using (III) on the way of searching templates using (II) (Sect. 4.4).

- The previous paper [1] evaluated our proposal using the CASIA-FingerprintV5 [17], the dataset of multiple fingerprints. Added to this, this paper also evaluates our proposal using the NIST BSSR1 Set3 [18], the dataset of multiple matchers (Sect. 5).

This paper is organized as follows. Section 2 describes the problems with the accuracy and response time in biometric identification and several approaches to the problems, explaining why we focus on score level fusion schemes and metric space indexing schemes. Section 3 introduces the previous work on the above schemes. Section 4 proposes our framework and the NBS scheme, and discuss the optimality of the NBS scheme. Section 5 shows the experimental results using the two datasets. Finally, Sect. 6 concludes this paper with directions for the future.

\section{Biometric Identification}

\subsection{Two Problems in Biometric Identification}

As described in Sect. 1, the two problems grow bigger as the number of enrollees increases: (1) the identification error rate increases; (2) the response time also increases. This section explains the problems more in detail.

Two kinds of error rates are defined in verification: FRR (False Reject Rate) and FAR (False Accept Rate). FRR is the probability that a genuine individual is rejected as an impostor, while FAR is the probability that an impostor is accepted as a genuine individual. Similarly, $\operatorname{FRR}(N)$ and $\operatorname{FAR}(N)$ are defined in identification [2], where $N$ is the number of enrollees. $\operatorname{FRR}(N)$ is the probability that an enrollee is rejected as a non-enrollee, while $\operatorname{FAR}(N)$ is the probability that a non-enrollee is accepted as some enrollee.

Let us assume that all scores are independent, and consider the following decision rule: if one or more scores (defined as similarities) exceed the threshold, accept the claimant as the enrollee whose score is the highest; otherwise, reject. Then, since the probability that a score between the different individuals exceeds the threshold is FAR, the following approximation can be obtained if $N \times \mathrm{FAR} \ll 1$ :

$$
\begin{aligned}
\operatorname{FAR}(N) & =1-(1-\mathrm{FAR})^{N} \\
& \approx N \times \mathrm{FAR} .
\end{aligned}
$$

That is, $\operatorname{FAR}(N)$ increases almost in proportion to $N$.

The one-to-many matching time also increases in proportion to $N$. For example, when a score level fusion scheme which combines scores from $M$ modalities is used to improve the accuracy, the system is required to compute $M N$ scores in total.

Thus, both $\operatorname{FAR}(N)$ and response time can be expressed as $\mathrm{O}(N)$, which makes biometric identification quite challenging to apply to the large scale applications.

\subsection{How Can We Solve the Two Problems?}

There are several approaches to improving the accuracy in biometric identification: (a) improving modules such as (a1) sensors, (a2) feature extractors, and (a3) matchers; (b) using multimodal fusion schemes [4]. Multimodal fusion schemes combine multiple modalities to recognize an individual with high accuracy, and can be classified into the following three categories [4]: (b1) feature level fusion schemes, (b2) score level fusion schemes, and (b3) decision level fusion schemes. They combine the multiple biometric features, scores, and final outputs (decision results), respectively.

Approaches to improving the response time in biometric identification are as follows: (i) improving matchers; (ii) using classification/indexing schemes at the feature level[19]-[24]; (iii) using metric space indexing schemes [5], [6]. The first approach reduces the one-to-one matching time (time to compute a score between one query sample and one template), while the second and third approaches reduce the number of score computations. Metric space indexing schemes have been developed in the area of similarity search, where the system outputs the objects (e.g. images, movies, or documents) in the database which are similar to the query object presented by the user. They reduce the number of score computations between the query 


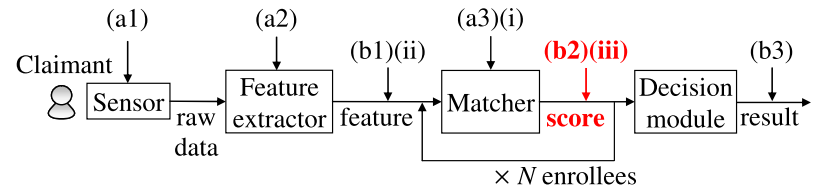

Fig. 1 Several approaches to improving the accuracy or response time in biometric identification ((a1)-(a3): improvement, (b1)-(b3): fusion, (i): improvement, (ii): classification/indexing, (iii) indexing).

object and the objects in the database using only scores between the objects as a clue. Thus, they can be applied to biometric identification as a speed-up technique at the score level.

Figure 1 shows the approaches to improving the accuracy or response time in biometric identification. Among them, improvement of modules (i.e. (a1), (a2), (a3), and (i)) and feature level approaches (i.e. (b1) and (ii)) generally specialize in a certain modality such as fingerprint, face, and iris, and do not have general applicability: they may not be able to be applied to other modalities. They also cannot be applied to most commercial biometric systems because they do not provide access to the features (nor raw data) [4]. In contrast, decision level fusion schemes (i.e. (b3)) can be applied to any kind of biometric system that outputs identification results. However, they are inferior to feature level fusion schemes and score level fusion schemes (i.e. (b1) and (b2)) in accuracy because identification results are less informative than features and scores.

Taking these matters into account, we focus on score level fusion schemes and metric space indexing schemes (i.e. (b2) and (iii)). Both of them only use scores, which are more informative than decision results, as information sources, and can be applied to any kind of biometric system that outputs scores.

\section{Related Work}

In this section, we introduce the previous work on score level fusion and metric space indexing. Although a number of schemes have been proposed in both of the area [4][6], this paper focuses on some specific schemes: the NBF (Naive Bayes Fusion) scheme [11] as a score level fusion scheme; the standard pivot-based indexing scheme [7] and the permutation-based indexing scheme [7], [8] as metric space indexing schemes. We first clarify the reason for focusing on them in Sects. 3.1 and 3.2. We then move on to pseudo-score based indexing schemes which include the above two indexing schemes, and provide a new, generalized framework in Sect.3.3.1 to describe them in a unified point of view. We finally explain the algorithm of the above two indexing schemes in Sects. 3.3.2 and 3.3.3.

\subsection{Score Level Fusion}

A number of score level fusion schemes have been proposed to improve the accuracy [4]. Some recently proposed schemes can also handle missing scores due to missing templates, missing query samples, or incomplete score lists [11]-[16]. In this paper, we make an attempt to combine a score level fusion scheme and a metric space indexing scheme, as described in Sect. 1. Then, since a metric space indexing scheme speeds up searching by reducing the number of score computations, we have to use a score level fusion scheme which can handle missing scores.

Among the above schemes, we focus on the score level fusion scheme using the Naive Bayes classifier, proposed by Nandakumar et al. [11], and use it in our experiments in Sect.5. In this paper, we refer to this scheme as the NBF (Naive Bayes Fusion) scheme. There are three reasons for using the NBF scheme: (1) The Bayes decision rule, which the Naive Bayes classifier adopts along with the assumption of independence of variables, minimizes the identification error rate in multi-class classification by selecting the class whose posterior probability of being a correct answer is the highest [25]. That is, the NBF scheme is optimal with regard to the accuracy if the posterior probability is perfectly estimated; (2) The NBF scheme is designed in the identification scenario [11]. On the other hand, since the schemes in [13]-[16] are designed in the verification scenario, they need to be extended to the identification scenario; (3) The NBF scheme is easy to implement.

The NBF scheme computes the posterior probability that the claimant is the $i$-th enrollee $(1 \leq i \leq N)$ (or a non-enrollee) using scores, and identifies the claimant by comparing it to the threshold. It can also handle missing scores by setting the corresponding likelihood ratio to 1 (or equivalently, the corresponding log-likelihood ratio to 0 ), as described in [11]. The details of the algorithm of the BSF scheme are described in Sect. 4.3.

\subsection{Metric Space Indexing}

Metric space indexing schemes have been developed in the area of similarity search [5], [6] to reduce the number of score computations at query time. Here, the goal is to quickly find a set of objects in the database whose distance (or similarity) to the query object is less than (or more than) the threshold, or to quickly find $K$ objects in the database closest to the query object (i.e. the answer of range queries or $K$-NN queries). A few studies applied metric space indexing schemes to biometrics [26]-[28].

These schemes can be divided into the following categories: exact indexing schemes and approximate (or inexact) indexing schemes. Exact indexing schemes make use of the triangle inequality to discard the objects which are not in the correct answer. The advantage of them is that they are guaranteed to output the correct answer. However, they suffer from the curse of dimensionality [5]: the triangle inequality is effective only in the case where the features are in low dimensional spaces, and they end up computing almost all scores in high dimensional spaces. Biometric features are often in high dimensional spaces (e.g. Daugman [29] used 2048-bit iriscodes) and distance measures in biometrics often do not satisfy the triangle-inequality. Thus, it is not often 
that the exact indexing schemes are useful in biometrics. Approximate indexing schemes are designed to quickly find an approximate answer even in the above cases. The performance of them is measured by the probability that the system fails to compute a score for an object in the correct answer and the percentage of the number of scores which are computed. In this paper, we refer to them as the retrieval error rate and the penetration rate, in a similar way to [19], respectively.

To date, a number of approximate indexing schemes have been proposed so far [6]. Among them, we focus on pseudo-score based indexing schemes [7]-[10], and use the standard pivot-based indexing scheme [7] and the permutation-based indexing scheme [7], [8] in our experiments in Sect. 5. There are two reasons for this: (1) Both of them provide a very good trade-off between the retrieval error rate and the penetration rate (i.e. they are state-of-theart schemes); (2) Both of them are easy to implement.

In the next subsection, we first explain the framework of pseudo-score based indexing schemes, and then explain the algorithm of the standard pivot-based indexing scheme [7] and the permutation-based indexing scheme [7], [8].

\subsection{Pseudo-Score Based Indexing}

\subsubsection{Framework}

Pseudo-score based indexing schemes [7]-[10] are approximate indexing schemes which reduce the number of score computations using pseudo-scores as a clue. Their examples include the standard pivot-based indexing scheme [7], the permutation-based indexing scheme [7], [8], the distance regression scheme [9], and BoostMAP [10]. Although they are independently proposed, in this paper we provide a new, generalized framework of pseudo-score based indexing schemes to describe them in a unified point of view.

We now explain the framework of pseudo-score based indexing schemes. Let $\mathbb{X}$ be a universe of objects, $q \in \mathbb{X}$ be a query object, and $\mathbb{O}=\left\{o_{i} \mid 1 \leq i \leq N\right\} \subset \mathbb{X}$ be a finite set of objects in the database. Let further $s: \mathbb{X} \times \mathbb{X} \rightarrow \mathbb{R}$ be a score function, and $\tilde{s}: \mathbb{X} \times \mathbb{X} \rightarrow \mathbb{R}$ be a pseudo-score function. To begin with, they construct an index $\mathbb{I}$ in advance, which is used to compute pseudo-scores at query time, as follows:

1. Select $P(<N)$ objects from $\mathbb{O}$ (at random [7], for example). The selected objects are referred to as pivots, and the remaining objects are referred to as non-pivots. We assume that $\mathbb{O}_{\text {piv }}=\left\{o_{i} \mid 1 \leq i \leq P\right\}$ is a set of pivots and $\mathbb{O}_{\text {non }}=\left\{o_{i} \mid P+1 \leq i \leq N\right\}$ is a set of non-pivots, without loss of generality.

2. Compute a score $s\left(o_{i}, o_{j}\right)$ for each pivot $o_{i} \in \mathbb{O}_{p i v}$ and each non-pivot $o_{j} \in \mathbb{O}_{\text {non }}$, and obtain a score matrix $\mathbb{S}_{\text {mat }}=\left\{s\left(o_{i}, o_{j}\right) \mid o_{i} \in \mathbb{O}_{\text {piv }}, o_{j} \in \mathbb{O}_{\text {non }}\right\}(P(N-P)$ scores in total).

3. Construct an index $\mathbb{I}$ using $\mathbb{S}_{\text {mat }}$, and store it in the database (the example of $\mathbb{I}$ is described later).
Then, they carry out the search at query time as follows:

1. Compute a score $s\left(q, o_{i}\right)$ for each pivot $o_{i} \in \mathbb{O}_{p i v}$, and obtain $\mathbb{S}_{\text {piv }}=\left\{s\left(q, o_{i}\right) \mid o_{i} \in \mathbb{O}_{\text {piv }}\right\}$ ( $P$ scores in total).

2. Compute a pseudo-score $\tilde{s}\left(q, o_{i}\right)$ for each non-pivot $o_{i} \in \mathbb{O}_{\text {non }}$ using $\mathbb{S}_{p i v}$ and $\mathbb{I}$ (the example of $\tilde{s}\left(q, o_{i}\right)$ is described later), and obtain $\tilde{\mathbb{S}}_{\text {non }}=\left\{\tilde{s}\left(q, o_{i}\right) \mid o_{i} \in \mathbb{O}_{\text {non }}\right\}$ $(N-P$ pseudo-scores in total).

3. Compute scores for $T(\leq N-P)$ non-pivots in ascending order of pseudo-scores.

4. Output the answer of range queries or $K$-NN queries.

That is, they reduce the number of score computations between the query object and the non-pivots from $N-P$ to $T$, by searching $T$ non-pivots in ascending order of pseudoscores, and stopping searching after that. They can control the trade-off between the retrieval error rate and the penetration rate by changing the value of $T$.

\subsubsection{Standard Pivot-Based Indexing Scheme}

The standard pivot-based indexing scheme, proposed by Chávez et al. [7], is the most basic type of pseudo-score based indexing scheme. We now explain the algorithm of this scheme, by clarifying the index $\mathbb{I}$ and the pseudo-score $\tilde{s}\left(q, o_{i}\right)$ in the above framework.

Let $\Phi: \mathbb{X} \rightarrow \mathbb{R}^{P}$ be a score vector function which maps the object $x \in \mathbb{X}$ to a score vector $\left(s\left(x, o_{1}\right), \cdots, s\left(x, o_{P}\right)\right)$ which is composed of scores for pivots. In the step 3 ) at indexing time, this scheme simply stores the score matrix $\mathbb{S}_{\text {mat }}$ in the database as an index. The index can then be expressed as a set of score vectors $\mathbb{I}=\left\{\Phi\left(o_{i}\right) \mid o_{i} \in \mathbb{O}_{\text {non }}\right\}$. In the step 2 ) at query time, it first generates a score vector $\Phi(q)$ from $\mathbb{S}_{p i v}$, and then computes, for each non-pivot $o_{i} \in \mathbb{O}_{\text {non }}$, the $L_{p}$ norm between $\Phi(q)$ and $\Phi\left(o_{i}\right)$ as a pseudo-score, which is given by

$$
L_{p}\left(\Phi(q), \Phi\left(o_{i}\right)\right)=\left(\sum_{j=1}^{P}\left(s\left(q, o_{j}\right)-s\left(o_{i}, o_{j}\right)\right)^{p}\right)^{1 / p} .
$$

This scheme can be regarded as a modification of LAESA (Linear Approximating and Eliminating Search Algorithm) [30] to use the general $L_{p}$ norm instead of $L_{\infty}$ in the approximating step and to skip the elimination step using the triangle inequality. In our experiments in Sect. 5, this scheme is denoted by $\mathbf{P} \mathbf{I}_{\Phi}$.

\subsubsection{Permutation-Based Indexing Scheme}

The permutation-based indexing scheme was independently proposed by Chávez et al. [7] and Amato and Savino [8]. This scheme computes a permutation, where the pivot IDs are written in ascending (or descending) order of distances (or similarities), and computes a distance between two permutations as a pseudo-score.

That is, in the step 3) at indexing time, this scheme computes, for each non-pivot $o_{i} \in \mathbb{O}_{\text {non }}$, a permutation $\Pi_{o_{i}}$ using $\mathbb{S}_{\text {mat }}$ (i.e. $\mathbb{I}=\left\{\Pi_{o_{i}} \mid o_{i} \in \mathbb{O}_{\text {non }}\right\}$ ). In the step 2) at query 


\begin{tabular}{|c|c|c|c|c|c|c|c|c|c|c|c|c|}
\hline & & \multicolumn{5}{|c|}{$\begin{array}{l}\text { Standard Pivot-based Indexing } \\
\text { Score Vector }\end{array}$} & & & $-\mathrm{P}$ & $\begin{array}{l}\text { rmu } \\
\text { tatio } \\
3_{\text {rd }}\end{array}$ & $\begin{array}{l}\text { tati } \\
\text { on } \\
4_{\text {th }}\end{array}$ & n-based Indexing \\
\hline & $\Phi(q)$ & 25 & 3 & 18 & 7 & & $\Pi_{q}$ & \begin{tabular}{|l|l}
$o_{2}$ \\
\end{tabular} & $o_{4}$ & $o_{3}$ & $o_{1}$ & \\
\hline & & & 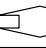 & E- & Ps & do-score $\left(\mathrm{L}_{1}\right.$ norm $)$ & & & 2 & E्. & - Ps & 10) \\
\hline & $\Phi\left(o_{5}\right)$ & 4 & 22 & 24 & 7 & $21+19+6+0=46$ & $\Pi_{o_{5}}$ & $o_{1}$ & $o_{4}$ & $o_{2}$ & $o_{3}$ & $3^{2}+2^{2}+1^{2}+0^{2}=14$ \\
\hline & $\Phi\left(o_{6}\right)$ & 20 & 8 & 16 & 5 & $5+5+2+2=14$ & $\Pi_{o_{6}}$ & $o_{4}$ & $o_{2}$ & $o_{3}$ & $o_{1}$ & $0^{2}+1^{2}+0^{2}+1^{2}=2$ \\
\hline$\because-o_{3}$ & $\Phi\left(o_{7}\right)$ & 25 & 18 & 4 & 15 & $0+15+14+8=37$ & $\Pi_{o_{7}}^{\circ}$ & $o_{3}$ & $o_{4}$ & $o_{2}$ & $o_{I}$ & $0^{2}+2^{2}+2^{2}+0^{2}=8$ \\
\hline & $\Phi\left(o_{8}\right)$ & 9 & 22 & 12 & 10 & $16+19+6+3=44$ & $\Pi_{o_{8}}$ & $o_{1}$ & $o_{4}$ & $o_{3}$ & $o_{2}$ & $3^{2}+3^{2}+0^{2}+0^{2}=18$ \\
\hline $\begin{array}{l}- \text { : query object }(q) \\
0: \text { pivot }(o, o)\end{array}$ & $\Phi\left(o_{9}\right)$ & 27 & 7 & 33 & 12 & $2+4+15+5=26$ & $\prod_{o_{9}}$ & \begin{tabular}{|l|}
$\mathrm{o}_{2}$ \\
\end{tabular} & $o_{4}$ & $o_{1}$ & $o_{3}$ & $1^{2}+0^{2}+1^{2}+0^{2}=2$ \\
\hline $0:$ non-pivot $\left(o_{5}-o_{10}\right)$ & $\Phi\left(o_{10}\right)$ & 14 & 11 & 28 & 6 & $11+8+10+1=30$ & $\Pi_{o_{10}}$ & $o_{4}$ & $o_{2}$ & $o_{1}$ & $o_{3}$ & $1^{2+}+1^{2}+1^{2}+1^{2}=4$ \\
\hline & & & Ind & & & & & & & & & \\
\hline
\end{tabular}

Fig. 2 Example of the standard pivot-based indexing scheme and the permutation-based indexing scheme $(P=4, N=10)$. The former scheme computes a score vector for each non-pivot as an index in advance. At query time, it first computes a score for each pivot, and then computes scores for $T$ $(\leq N-P=6)$ non-pivots in ascending order of pseudo-scores (in this case, in an order of $o_{6}, o_{9}, o_{10}$, $\cdots$ ). The latter scheme uses permutations instead of score vectors.

time, it first computes a permutation $\Pi_{q}$ for the query object $q \in \mathbb{X}$ using $\mathbb{S}_{p i v}$, and then computes, for each non-pivot $o_{i} \in \mathbb{O}_{\text {non }}$, a distance between $\Pi_{q}$ and $\Pi_{o_{i}}$ as a pseudo-score.

As a distance measure between two permutations, the rank correlation such as Spearman's Footrule, Spearman's Rho, and Kendall Tau can be used [7]. For example, Spearman's Rho can be expressed as

$$
S_{\rho}\left(\Pi_{q}, \Pi_{o_{i}}\right)=\sum_{j=1}^{P}\left(\Pi_{q}^{-1}(j)-\Pi_{o_{i}}^{-1}(j)\right)^{2},
$$

where $\Pi^{-1}(j)$ denotes the position of $o_{j}$ in $\Pi[7]$. For example, if $\Pi_{q}=o_{2}, o_{4}, o_{3}, o_{1}$ and $\Pi_{o_{i}}=o_{1}, o_{4}, o_{2}, o_{3}$, then $S_{\rho}\left(\Pi_{q}, \Pi_{o_{i}}\right)=(4-1)^{2}+(1-3)^{2}+(3-4)^{2}+(2-2)^{2}=14$. In Sect. 5, this scheme is denoted by $\mathbf{P I}_{\Pi}$.

Figure 2 shows the example of the standard pivotbased indexing scheme and the permutation-based indexing scheme, in the case where $P=4, N=10$, and the $L_{1}$ norm and Spearman's Rho are used as a distance measure between two score vectors and two permutations, respectively.

\section{A General Framework and Algorithms for Score Level Indexing and Fusion}

In this section, we first propose a score level indexing and fusion framework which can be constructed from (I) a pseudo-score based indexing scheme [7]-[10], (II) a multibiometric search scheme, and (III) a score level fusion scheme which handles missing scores [11]-[16]. We can use any existing scheme as (I) or (III), while (II) is a new scheme which is necessary to construct our framework. We begin with the overview of our framework.

Figure 3 shows the flowchart of the authentication process in our framework, where $M$ is the number of modalities. In our framework, (I) a pseudo-score based indexing scheme computes, for each modality, scores for $P$ pivots and pseudo-scores for $N-P$ non-pivots. Then, (II) a multi-biometric search scheme computes scores for $T$

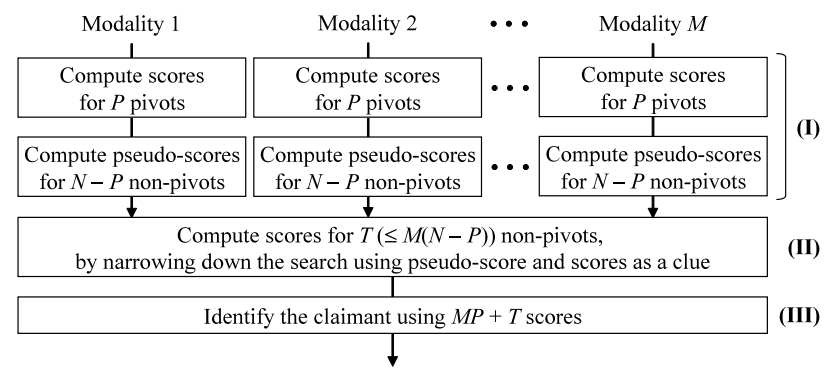

Fig. 3 Flowchart of the authentication process in our framework ((I): pseudo-score based indexing scheme, (II): multi-biometric search scheme, (III): score level fusion scheme which handles missing scores).

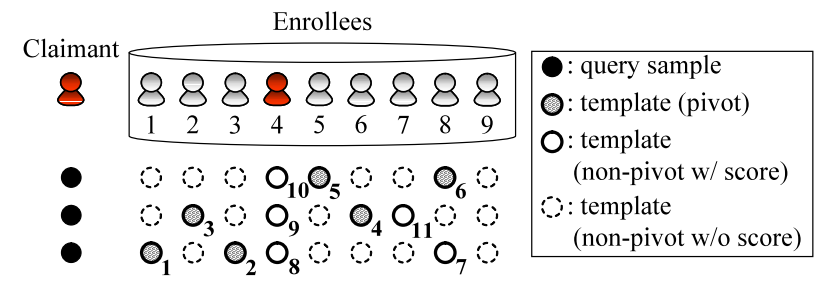

Fig. 4 Example of the order of score computations in our framework ( $N=9, P=2, M=3, T=5$; the claimant is the 4th enrollee). Numbers besides the templates represent the order of score computations. Scores for non-pivots denoted by dashed circles are missing.

$(\leq M(N-P))$ non-pivots, by narrowing down the search using pseudo-scores and scores as a clue. Finally, (III) a score level fusion scheme identifies a claimant using $M P+T$ scores (i.e. $M P$ scores for pivots and $T$ scores for nonpivots; $M N-(M P+T)$ scores are missing). It improves the response time by reducing the number of score computations from $M N$ to $M P+T$ ( $\leq M N$ ) using (II), and improves the accuracy by combining scores from $M$ modalities using (III).

Figure 4 shows the example of the order of score computations in the case where $N=9, P=2, M=3, T=5$, and the claimant is the 4th enrollee. As described in Sect. 3.3.1, pseudo-score based indexing schemes compute scores for 
non-pivots in ascending order of pseudo-scores. However, we emphasize that scores are also very useful in finding genuine templates in multi-biometric identification where there are more than one templates per enrollee. For example, if we find that the 8th score (defined as a similarity) in Fig. 4 is very high, we can expect that the claimant is the 4th enrollee. Then, since genuine scores play an important role to correctly identify the claimant, we should go on computing scores for the 4th enrollee, as illustrated in Fig. 4, to compute as many genuine scores as possible. Taking this matter into accounts, (II) the multi-biometric search scheme narrows down the search using not only pseudo-scores but scores. This is the most significant feature of (II). We show that the accuracy is significantly improved by doing so in our experiments in Sect. 5.

The remaining of this section is as follows. Section 4.1 explains our framework in detail. Section 4.2 proposes the NBS (Naive Bayes Search) scheme as (II), and discusses its optimality with respect to the retrieval error rate (i.e. the probability that the system fails to compute a genuine score). Section 4.3 clarifies the remaining two component (i.e. (I) and (III)) in our framework. Section 4.4 proposes a technique which further reduces the average number of score computations without significantly affecting the accuracy.

\subsection{Framework}

We now explain our framework in detail, using the notations shown in Table 1. Assume the multi-biometric identification system which combines $M$ different biometric traits (e.g. fingerprint, face, and iris) or fingers (e.g. index and middle fingerprints). The discussion below can be easily extended to multiple matchers by assuming the same query sample and the same templates across different modalities. Let $\mathbb{X}$ be a universe of biometric samples, $\mathbb{Q}=\left\{q^{m} \mid 1 \leq m \leq M\right\} \subset \mathbb{X}$ be a set of query samples, where $q^{m}$ is a query sample from the $m$-th modality, and $\mathbb{O}=\left\{o_{i}^{m} \mid 1 \leq m \leq M, 1 \leq i \leq N\right\} \subset \mathbb{X}$ be a finite set of templates in the database, where $o_{i}^{m}$ is a

Table 1 Notations used in our framework.

\begin{tabular}{l|l}
\hline \hline Symbol & Description $(1 \leq i \leq N, 1 \leq m \leq M)$ \\
\hline$N$ & number of enrollees \\
$P$ & number of pivots per modality \\
$M$ & number of modalities \\
$T$ & number of score computations for non-pivots $(\leq M(N-P))$ \\
$P R_{\text {req }}$ & required penetration rate $(=(M P+T) / M N * 100[\%])$ \\
$\mathbb{X}$ & universe of biometric samples \\
$\mathbb{Q}$ & set of query samples in the database $\mathbb{Q} \subset \mathbb{X}$ \\
$\mathbb{O}^{-}$ & set of templates in the database $\mathbb{O} \subset \mathbb{X}$ \\
$\mathbb{O}_{\text {piv }}$ & set of pivots $\mathbb{O}_{\text {piv }} \subset \mathbb{O}$ \\
$\mathbb{O}_{\text {non }}$ & set of non-pivots $\mathbb{O}_{\text {non }}=\mathbb{O} \backslash \mathbb{O}_{\text {piv }}$ \\
$q^{m}$ & query sample from the $m$-th modality $q^{m} \in \mathbb{X}$ \\
$o_{i}^{m}$ & template of the $i$-th enrollee from the $m$-th modality $o_{i}^{m} \in \mathbb{O}$ \\
$s$ & score function $s: \mathbb{X} \times \mathbb{X} \rightarrow \mathbb{R}$ \\
$\tilde{s}$ & pseudo-score function $\tilde{s}: \mathbb{X} \times \mathbb{X} \rightarrow \mathbb{R}$ \\
$\mathbb{S}_{\text {piv }}$ & set of scores for $M P$ pivots \\
$\tilde{\mathbb{S}}_{\text {non }}$ & set of pseudo-scores for $M(N-P)$ non-pivots \\
$\mathbb{S}_{\text {non }}^{(t)}$ & set of scores for non-pivots after computing scores for $t$ non- \\
& pivots $(0 \leq t \leq T)$ \\
\hline
\end{tabular}

template of the $i$-th enrollee from the $m$-th modality. Let further $s: \mathbb{X} \times \mathbb{X} \rightarrow \mathbb{R}$ be a score function, and $\tilde{s}: \mathbb{X} \times \mathbb{X} \rightarrow \mathbb{R}$ be a pseudo-score function, in the same way as Sect. 3.3.

To begin with, our framework selects, for each modality, $P(<N)$ pivots from $N$ templates and constructs an index, in the same way as Sect. 3.3. Here, we can change enrollees who have pivots depending on modalities. Let $\mathbb{O}_{\text {piv }}$ be a set of pivots ( $M P$ pivots in total), and $\mathbb{O}_{\text {non }}$ be a set of non-pivots $(M(N-P)$ non-pivots in total). Let further $\mathbb{S}_{\text {piv }}=\left\{s\left(q^{m}, o_{i}^{m}\right) \mid q^{m} \in \mathbb{Q}, o_{i}^{m} \in \mathbb{O}_{\text {piv }}\right\}$ be a set of scores for all pivots, $\tilde{\mathbb{S}}_{\text {non }}=\left\{\tilde{s}\left(q^{m}, o_{i}^{m}\right) \mid q^{m} \in \mathbb{Q}, o_{i}^{m} \in \mathbb{O}_{\text {non }}\right\}$ be a set of pseudo-scores for all non-pivots, and $\mathbb{S}_{\text {non }}^{(t)}$ be a set of scores for non-pivots after computing scores for $t$ non-pivots $(0 \leq t \leq T)$.

After the claimant inputs all query samples, our framework carries out the one-to-many matching process and the identification process, as follows:

1. Compute a set of scores for all pivots $\mathbb{S}_{p i v}(M P$ scores in total).

2. Compute a set of pseudo-scores for all non-pivots $\tilde{\mathbb{S}}_{n o n}$ $(M(N-P)$ pseudo-scores in total).

3. $t \leftarrow 0$.

4. Select a non-pivot using a set of pseudo-scores and scores $\left\{\mathbb{S}_{\text {piv }}, \tilde{\mathbb{S}}_{n o n}, \mathbb{S}_{\text {non }}^{(t)}\right\}$ as a clue, and compute a score for the non-pivot.

5. $t \leftarrow t+1$. If $t<T$, go back to the step 3 .

6. Identify the claimant using a set of scores $\left\{\mathbb{S}_{p i v}, \mathbb{S}_{n o n}^{(T)}\right\}$ $(M P+T$ scores in total), and output the identification result (i.e. enrollee ID or "reject").

The step 1 and 2 are carried out using (I) a pseudo-score based indexing scheme, the step 3, 4 and 5 are carried out using (II) a multi-biometric search scheme, and the step 6 is carried out using (III) a score level fusion scheme which handles missing scores. Let $P R_{r e q}=(M P+T) / M N * 100[\%]$ be the required penetration rate. Then, since our framework computes $M P+T$ scores, it keeps the penetration rate to be $P R_{\text {req }}[\%]$ (i.e. it meets the requirement).

\subsection{Naive Bayes-Based Multi-Biometric Search}

\subsubsection{Algorithm}

The multi-biometric search scheme selects a non-pivot using a set of pseudo-scores and scores $\left\{\mathbb{S}_{\text {piv }}, \tilde{\mathbb{S}}_{n o n}, \mathbb{S}_{n o n}^{(t)}\right\}(0 \leq$ $t<T)$ as a clue. Then, it would be natural to consider that we should select the non-pivot whose posterior probability of being a genuine template is the highest to minimize the retrieval error rate.

The NBS (Naive Bayes Search) scheme is based on this idea. Let $H_{i}(0 \leq i \leq N)$ be the following hypothesis:

$H_{i}$ : The claimant is the $i$-th enrollee. $(1 \leq i \leq N)$

$H_{0}$ : The claimant is a non-enrollee.

Then, the NBS scheme selects the non-pivot of the enrollee whose posterior probability $P\left(H_{i} \mid \mathbb{S}_{p i v}, \widetilde{\mathbb{S}}_{n o n}, \mathbb{S}_{n o n}^{(t)}\right)(1 \leq i \leq$ $N)$ is the highest. $P\left(H_{i} \mid \mathbb{S}_{p i v}, \tilde{\mathbb{S}}_{n o n}, \mathbb{S}_{n o n}^{(t)}\right)$ can be written, using Bayes' theorem, as follows: 


$$
P\left(H_{i} \mid \mathbb{S}_{p i v}, \tilde{\mathbb{S}}_{n o n}, \mathbb{S}_{n o n}^{(t)}\right)=\frac{\exp \left(Z_{i}^{(t)}\right) P\left(H_{i}\right)}{\sum_{n=0}^{N} \exp \left(Z_{n}^{(t)}\right) P\left(H_{n}\right)},
$$

where $Z_{i}^{(t)}$ is the log-likelihood ratio which is given by

$$
Z_{i}^{(t)}=\log \frac{P\left(\mathbb{S}_{p i v}, \tilde{\mathbb{S}}_{n o n}, \mathbb{S}_{\text {non }}^{(t)} \mid H_{i}\right)}{P\left(\mathbb{S}_{p i v}, \tilde{\mathbb{S}}_{n o n}, \mathbb{S}_{n o n}^{(t)} \mid H_{0}\right)},
$$

and $P\left(H_{i}\right)$ is the prior probability that $H_{i}$ is true. Since it is generally very difficult to correctly estimate $P\left(H_{i}\right)$, we assume that $P\left(H_{i}\right)$ is uniform (i.e. $P\left(H_{0}\right)=\cdots=P\left(H_{N}\right)=$ $1 /(N+1))$, for example. This form of prior distribution is known as a noninformative prior which is intended to have little influence on the posterior distribution (i.e. to "let the data speak for themselves") [31]. Furthermore, it can be seen from (5) that if $P\left(H_{i}\right)$ is uniform, the enrollee whose $P\left(H_{i} \mid \mathbb{S}_{\text {piv }}, \tilde{\mathbb{S}}_{n o n}, \mathbb{S}_{n o n}^{(t)}\right)$ is the highest is the same person as the one whose $Z_{i}^{(t)}$ is the highest. Thus, we only have to compute $Z_{i}^{(t)}$ instead of $P\left(H_{i} \mid \mathbb{S}_{\text {piv }}, \tilde{\mathbb{S}}_{\text {non }}, \mathbb{S}_{\text {non }}^{(t)}\right)$ in such a case. In our experiments in Sect. 5, we set $P\left(H_{i}\right)$ to be uniform.

To simply compute $Z_{i}^{(t)}$, we assume that all scores and pseudo-scores are independent, and that genuine scores, impostor scores, genuine pseudo-scores, and impostor pseudoscores from the $m$-th modality are generated from $f_{g e n}^{m}()$, $f_{i m p}^{m}(), g_{g e n}^{m}()$, and $g_{i m p}^{m}()$, respectively. That is,

$$
\begin{aligned}
& \left.P\left(s\left(q^{m}, o_{i}^{m}\right) \mid H_{j}\right)=f_{\text {gen }}^{m}\left(s\left(q^{m}, o_{i}^{m}\right)\right) \quad \text { if } i=j\right), \\
& P\left(s\left(q^{m}, o_{i}^{m}\right) \mid H_{j}\right)=f_{i m p}^{m}\left(s\left(q^{m}, o_{i}^{m}\right)\right) \quad(\text { if } i \neq j), \\
& P\left(\tilde{s}\left(q^{m}, o_{i}^{m}\right) \mid H_{j}\right)=g_{g e n}^{m}\left(\tilde{s}\left(q^{m}, o_{i}^{m}\right)\right) \quad(\text { if } i=j), \\
& P\left(\tilde{s}\left(q^{m}, o_{i}^{m}\right) \mid H_{j}\right)=g_{i m p}^{m}\left(\tilde{s}\left(q^{m}, o_{i}^{m}\right)\right) \quad(\text { if } i \neq j) .
\end{aligned}
$$

Then, $Z_{i}^{(t)}$ in (6) can be decomposed as follows:

$$
Z_{i}^{(t)}= \begin{cases}0 & (\text { if } i=0) \\ \sum_{m=1}^{M} \alpha_{i}^{m^{(t)}} F^{m}\left(s\left(q^{m}, o_{i}^{m}\right)\right) & \\ +\sum_{m=1}^{M} \beta_{i}^{m} G^{m}\left(\tilde{s}\left(q^{m}, o_{i}^{m}\right)\right) & (\text { if } 1 \leq i \leq N),\end{cases}
$$

where $\alpha_{i}^{m^{(t)}} \in\{0,1\}$ is a value which takes 1 if $s\left(q^{m}, o_{i}^{m}\right)$ has been computed after searching $t$ non-pivots and 0 otherwise, $\beta_{i}^{m} \in\{0,1\}$ is a value which takes 1 if $\tilde{s}\left(q^{m}, o_{i}^{m}\right)$ has been computed (i.e. $o_{i}^{m}$ is a non-pivot) and 0 otherwise, and $F^{m}()$ and $G^{m}()$ are the $\log$-likelihood ratio functions given by

$$
\begin{aligned}
& F^{m}\left(s\left(q^{m}, o_{i}^{m}\right)\right)=\log \frac{f_{\text {gen }}^{m}\left(s\left(q^{m}, o_{i}^{m}\right)\right)}{f_{i m p}^{m}\left(s\left(q^{m}, o_{i}^{m}\right)\right)}, \\
& G^{m}\left(\tilde{s}\left(q^{m}, o_{i}^{m}\right)\right)=\log \frac{g_{g e n}^{m}\left(\tilde{s}\left(q^{m}, o_{i}^{m}\right)\right)}{g_{i m p}^{m}\left(\tilde{s}\left(q^{m}, o_{i}^{m}\right)\right)} .
\end{aligned}
$$

Here, we assume the logistic regression model [31], which models $F^{m}()$ and $G^{m}()$ as a linear function of a score and a pseudo-score, respectively, as follows:

$$
\begin{aligned}
& F^{m}\left(s\left(q^{m}, o_{i}^{m}\right)\right)=w_{1}^{m} s\left(q^{m}, o_{i}^{m}\right)+w_{0}^{m}, \\
& G^{m}\left(\tilde{s}\left(q^{m}, o_{i}^{m}\right)\right)=\tilde{w}_{1}^{m} \tilde{s}\left(q^{m}, o_{i}^{m}\right)+\tilde{w}_{0}^{m},
\end{aligned}
$$

where $\left(w_{1}^{m}, w_{0}^{m}\right)$ and $\left(\tilde{w}_{1}^{m}, \tilde{w}_{0}^{m}\right)$ are regression coefficients of
$F^{m}()$ and $G^{m}()$, respectively. They are trained using biometric samples from enrollees or any other biometric samples collected in advance. There are two reasons for assuming the logistic regression model: (1) The validity of this model is shown in biometrics [12], [32]. We also demonstrate the validity of this model in our experiments in Sect. 5; (2) This model enables us to compute $F^{m}()$ and $G^{m}()$ with only one multiplication and one addition (see (14), (15)). Since $Z_{i}^{(t)}$ in (11) can be updated by adding the newly computed $F^{m}\left(s\left(q^{m}, o_{i}^{m}\right)\right)$ value to $Z_{i}^{(t-1)}$, we can compute $Z_{i}^{(t)}$ with only one multiplication and two additions, in total. Indeed, we confirmed that the time to update $Z_{i}^{(t)}$ was negligible, compared to the computation of a score, in Sect. 5 .

To sum up, if $P\left(H_{i}\right)(0 \leq i \leq N)$ is uniform, the algorithm of the NBS scheme can be written as follows:

1. $t \leftarrow 0$.

2. Compute $Z_{i}^{(t)}(1 \leq i \leq N)$ using (11), (14), and (15).

3. Select a non-pivot of the enrollee whose $Z_{i}^{(t)}$ is the highest among those who have one or more non-pivots with missing scores, and compute a score for the non-pivot.

4. $t \leftarrow t+1$. If $t<T$, go back to the step 2 .

In the step 3, if the selected enrollee has more than one nonpivots with missing scores, we need to select one of them. In Sect. 5, we evaluated $\operatorname{EER}(N)$ (Equal Error Rate; the operating point where $\operatorname{FAR}(N)=\operatorname{FRR}(N))$ [2] for each modality using the training samples, and selected the non-pivot whose $\operatorname{EER}(N)$ is the lowest, to improve the accuracy by starting score computations from the modality which captures the most discriminative information.

\subsubsection{Optimality}

We now discuss the optimality of the NBS scheme with regard to the retrieval error rate. We first assume that we can change the multi-biometric search scheme at each step to select a non-pivot (i.e. we can use a certain scheme in selecting the first non-pivot, and use a different scheme in selecting the second non-pivot, and so on). We refer to the $t$-th step to select a non-pivot simply as a $t$-th step. Then, we can prove the following lemma:

Lemma 1: Fix a multi-biometric search scheme at each step from the first to the (t-1)-th step. Then, if the posterior probability $P\left(H_{i} \mid \mathbb{S}_{\text {piv }}, \tilde{\mathbb{S}}_{n o n}, \mathbb{S}_{n o n}^{(t-1)}\right)(0 \leq i \leq N)$ can be perfectly estimated for any $\left\{\mathbb{S}_{\text {piv }}, \tilde{\mathbb{S}}_{n o n}, \mathbb{S}_{\text {non }}^{(t-1)}\right\}$, the NBS scheme minimizes the retrieval error rate at the $t$-th step (i.e. the probability that the $t$-th non-pivot is not a genuine template even as the claimant is an enrollee) among all multi-biometric search schemes.

Proof: Let $U^{(t)} \in\{1,2, \cdots, N\}$ be an enrollee ID corresponding to the $t$-th non-pivot, and $\mathbb{T}^{(t-1)}=\left\{\mathbb{S}_{\text {piv }}, \tilde{\mathbb{S}}_{\text {non }}, \mathbb{S}_{\text {non }}^{(t-1)}\right\}$ be a set of pseudo-scores and scores obtained after the $(t-1)$ th step. Then, since the retrieval error rate at the $t$-th step is the probability that the claimant is neither $U^{(t)}$ nor a nonenrollee, it can be written as follows: 


$$
\begin{aligned}
& 1-P\left(H_{0}\right)-P\left(H_{U^{(t)}}\right) \\
= & 1-P\left(H_{0}\right)-\int P\left(H_{U^{(t)}} \mid \mathbb{T}^{(t-1)}\right) P\left(\mathbb{T}^{(t-1)}\right) d \mathbb{T}^{(t-1)} .
\end{aligned}
$$

Since we fix a multi-biometric search scheme at each step from the first to the $(t-1)$-th step, the prior probability $P\left(\mathbb{T}^{(t-1)}\right)$ takes a certain fixed value for any $\mathbb{T}^{(t-1)}$. Thus, if we maximize $P\left(H_{U^{(t)}} \mid \mathbb{T}^{(t-1)}\right)$ for any $\mathbb{T}^{(t-1)}$, then we can minimize the retrieval error rate in (17). This can be achieved when we use the NBS scheme which selects the enrollee whose posterior probability is the highest, if $P\left(H_{i} \mid \mathbb{T}^{(t-1)}\right)$ can be perfectly estimated for any $\mathbb{T}^{(t-1)}$.

This lemma states that if we fix a multi-biometric search scheme (or schemes) to select $t-1$ non-pivots, then the NBS scheme which perfectly estimates the posterior probability minimizes the retrieval error rate at the $t$-th step. However, it should be noted that we just proved the optimality of the NBS scheme in a limited sense. For example, we can say that the NBS scheme minimizes the retrieval error rate at the first step. We can also say that this scheme minimizes the retrieval error rate at the $t$-th step, under the condition that we use a certain, fixed scheme (or schemes) until the $(t-1)$-step. However, we cannot say that continuing to use the NBS scheme from the first to the $t$-th step is equivalent to or better than continuing to use any other multi-biometric search schemes from the first to the $t$-th step, because the schemes until the $(t-1)$-th step are different in this case (and hence the prior distributions of $\mathbb{T}^{(t-1)}$ are different).

Furthermore, it is extremely difficult to perfectly estimate the posterior probability $P\left(H_{i} \mid \mathbb{S}_{p i v}, \tilde{\mathbb{S}}_{n o n}, \mathbb{S}_{n o n}^{(t)}\right)$ in reality. Especially, although we assumed that all scores and pseudo-scores were independent to simply compute the loglikelihood ratio $Z_{i}^{(t)}$ (i.e. to compute $Z_{i}^{(t)}$ with only one multiplication and two additions), this is not correct to be exact because pseudo-scores are designed to be relevant to scores. The estimation of $Z_{i}^{(t)}$ which takes into account the correlation between scores and pseudo-scores is left as future work.

Scores across different modalities are also highly correlated in the case of multiple matchers. However, Nandakumar et al. [33] showed that the assumption of independence between matchers did not adversely affect the performance, especially when the individuals matchers are accurate and the difference between genuine and impostor correlation is not high. In our experiments in Sect.5, we evaluated our proposals in the multiple matchers scenario, using the NIST BSSR 1 Set3 which was also used in [33].

\subsection{The Other Two Components in Our Framework}

We also clarify the remaining two components which construct our framework.

\subsubsection{Pseudo-Score Based Indexing}

In our experiments in Sect. 5, we used the standard pivotbased indexing scheme [7] and the permutation-based indexing scheme [7], [8] as a pseudo-score based indexing scheme. The index was a set of score vectors $\mathbb{I}=$ $\left\{\Phi\left(o_{i}^{m}\right) \mid o_{i}^{m} \in \mathbb{O}_{\text {non }}\right\}(M(N-P)$ score vectors in total $)$ and a set of permutations $\mathbb{I}=\left\{\Pi_{o_{i}^{m}} \mid o_{i}^{m} \in \mathbb{O}_{\text {non }}\right\}(M(N-P)$ permutations in total), respectively.

\subsubsection{Score Level Fusion Which Handles Missing Scores}

As for a score level fusion scheme, we used the NBF scheme [11] in Sect. 5. This scheme computes the posterior probability $P\left(H_{i} \mid \mathbb{S}_{p i v}, \mathbb{S}_{\text {non }}^{(T)}\right)(0 \leq i \leq N)$, and identifies the claimant as follows: if the maximum of $P\left(H_{i} \mid \mathbb{S}_{\text {piv }}, \mathbb{S}_{\text {non }}^{(T)}\right)$ exceeds the identification threshold $A$, accept the corresponding hypothesis $H_{i}$ (if $i=0$, reject the claimant); otherwise, reject. Although [11] did not compute the posterior probability that $H_{0}$ is true (i.e. the claimant is a non-enrollee), we computed that in a similar way to [12].

The posterior probability $P\left(H_{i} \mid \mathbb{S}_{\text {piv }}, \mathbb{S}_{\text {non }}^{(T)}\right)$ can be written, in a similar way to Sect. 4.2.1, as follows:

$$
P\left(H_{i} \mid \mathbb{S}_{p i v}, \mathbb{S}_{n o n}^{(T)}\right)=\frac{\exp \left(Z_{i}^{\prime(T)}\right) P\left(H_{i}\right)}{\sum_{n=0}^{N} \exp \left(Z_{n}^{\prime(T)}\right) P\left(H_{n}\right)},
$$

where

$$
Z_{i}^{\prime(T)}= \begin{cases}0 & \text { (if } i=0) \\ \sum_{m=1}^{M} \alpha_{i}^{m^{(T)}} F^{m}\left(s\left(q^{m}, o_{i}^{m}\right)\right) & (\text { if } 1 \leq i \leq N) .\end{cases}
$$

Here we can see that missing scores are handled by setting the corresponding log-likelihood ratio to be 0 (i.e. $\alpha_{i}^{m^{(T)}}=$ 0), as described in Sect. 3.1.

\subsection{Reducing the Average Number of Score Computations}

In our framework in Sect. 4.1, (II) a multi-biometric search scheme computes scores for $T(\leq M(N-P))$ non-pivots, where $T$ is a predetermined value. In this paper, we also propose a technique for further reducing the average number of score computations, without significantly affecting the accuracy. This technique identifies the claimant using (III) a score level fusion scheme on the way of searching $T$ nonpivots using (II) a multi-biometric search scheme. That is, we change the step 4 in Sect. 4.1 as follows:

4-1. Identify the claimant using a set of scores $\left\{\mathbb{S}_{p i v}, \mathbb{S}_{n o n}^{(t)}\right\}$ $(M P+t$ scores in total). If the claimant is identified as some enrollee or a non-enrollee, output the identification result (i.e. enrollee ID or "reject") and terminate the process. Otherwise, go to the step 4-2.

4-2. Select a non-pivot using a set of pseudo-scores and scores $\left\{\mathbb{S}_{p i v}, \widetilde{\mathbb{S}}_{n o n}, \mathbb{S}_{n o n}^{(t)}\right\}$ as a clue, and compute a score for the non-pivot.

The step 4-1 is carried out using (III) a score level fusion scheme, and the step 4-2 is carried out using (II) a multibiometric search scheme. For example, in the case where the NBF scheme is used as (III), the step 4-1 can be written as follows: if the maximum of $P\left(H_{i} \mid \mathbb{S}_{p i v}, \mathbb{S}_{n o n}^{(t)}\right)$ exceeds the identification threshold $A$, accept the corresponding hypothesis $H_{i}$; otherwise, go to the step 4-2. That is, the proposed 
technique terminates the process on the way of searching non-pivots iff it finds out who the claimant is with enough confidence. By doing so, we can reduce the average number of score computations without significantly affecting the accuracy.

Since the step 4-1 may cause overhead, we do not have to carry out the step 4-1 every time. In our experiments in Sect. 5, we carried out the step 4-1 at the intervals of computing scores for 10 non-pivots, and confirmed that there was no overhead.

\section{Experimental Evaluation}

\subsection{Experimental Set-Up}

We evaluated our proposal using the CASIAFingerprintV5 [17] and the NIST BSSR1 Set3 [18], the dataset of multiple fingerprint images and face scores from the multiple matchers, respectively. There are two reasons for using these datasets: (1) We could obtain more than one score matrix per modality in these datasets, one of which is necessary to construct an index, and another is necessary to evaluate the performance; (2) They are relatively large datasets. For example, many papers evaluated their indexing methods using the FVC dataset which only contains images from 100 fingers [20]-[23]. In contrast, the CASIAFingerprintV5 and the NIST BSSR1 Set 3 contain 500 subjects and 3000 subjects, respectively.

In the next subsection, we explain the methods we evaluated. Then, we explain the details of the two datasets.

\subsubsection{Methods}

In our experiments, we evaluated the performance of the following schemes:

1. UB denotes the unimodal biometric scheme which computes scores for all templates from a single modality ( $N$ scores in total), and then identifies a claimant as follows: if one or more scores (defined as similarities) exceed the threshold, identify the claimant as the enrollee whose score is the highest; otherwise, reject.

2. $\mathbf{P I}_{\Phi}-\mathbf{N B F}$ denotes a simple combination of the standard pivot-based indexing scheme $\mathbf{P I}_{\Phi}$ and the NBF scheme. That is, after computing scores for all $M P$ pivots, it selects $T$ non-pivots from $M(N-P)$ non-pivots in ascending order of pseudo-scores and computes scores for them $(M P+T(\leq M N)$ scores in total). Finally, it identifies the claimant using the NBF scheme.

3. $\mathbf{P} \mathbf{I}_{\Pi}-\mathbf{N B F}$ is identical to $\mathbf{P I}_{\Phi}-\mathbf{N B F}$ except that it uses the permutation-based indexing scheme $\mathbf{P} \mathbf{I}_{\Pi}$.

4. $\mathbf{P I}_{\Phi}-\mathbf{N B S}-\mathbf{N B F}$ denotes our proposal which uses $\mathbf{P I}_{\Phi}$, the NBS scheme, and the NBF scheme. It also uses the technique for reducing the average number of score computations (described in Sect. 4.4) in evaluating the identification performance (see Sect. 5.2.3) (at most $M P+T(\leq M N)$ scores in total).

\section{5. $\mathbf{P I}_{\Pi}-\mathbf{N B S}-\mathbf{N B F}$ is identical to $\mathbf{P I}_{\Phi}-\mathbf{N B S}-\mathbf{N B F}$ except that it uses $\mathbf{P} \mathbf{I}_{\Pi}$.}

We evaluated $\mathbf{U B}, \mathbf{P I}_{\Phi}-\mathbf{N B F}$ and $\mathbf{P I}_{\Phi}-\mathbf{N B F}$ to demonstrate the effectiveness of our proposal through the comparison with them. In $\mathbf{P \mathbf { I } _ { \Phi }}$ and $\mathbf{P} \mathbf{I}_{\Pi}$, we used the $L_{1}$ norm and Spearman's Rho as a distance measure between two score vectors and two permutations, respectively. In NBS and NBF, we set the prior probability $P\left(H_{i}\right)$ to be uniform (i.e. $\left.P\left(H_{0}\right)=\cdots=P\left(H_{N}\right)=1 /(N+1)\right)$ and computed the loglikelihood ratio functions $F^{m}()$ and $G^{m}()$ using the logistic regression model (the way of training the regression coefficients is described in Sects. 5.1.2 and 5.1.3).

As for the number of pivots, 32, 64, 128, or 256 objects were selected as pivots from hundreds or tens of thousands of objects in the database in [7]. Since the number of subjects was 500 or 3000 in the datasets of our experiments, we selected, for each modality, 32 templates as pivots. Automatic determination of the optimal number of pivots with respect to the retrieval error rate is left as future work.

As a way of selecting pivots, we used the random selection method [7] which randomly selects pivots from the database, for each modality. The reason for using this method is that it provided the performance comparable or better than other selection methods in the experiments of [7], in spite of simpleness of the method (i.e. low computational complexity in selecting pivots).

\subsubsection{CASIA-FingerprintV5}

The first dataset is the CASIA-FingerprintV5 [17] which contains 20000 fingerprint images (left and right thumb/ index/middle/ring finger) from 500 subjects (each subject contributed 5 samples per finger). We selected one sample per finger as a template and computed scores using SourceAFIS Version 1.4 [34]. We used scores of left index, left middle, right index, and right middle finger $(M=4)$ $(4 \times 500 \times 500 \times 5$ scores in total, including scores between templates).

We randomly divided 500 subjects into 450 enrollees and 50 non-enrollees, and randomly selected 32 pivots per modality $(N=450, P=32)$. Here, we randomly chose 10 ways to select enrollees and pivots, to stabilize the performance we evaluated. Then, we further divided 4 samples of each enrollee (except the template) into one training sample and three testing samples (i.e. query samples). As for nonenrollees, we used all the 5 samples as testing samples, to evaluate the performance using as many testing samples as possible. The number of templates, training samples, and testing samples was 450,450 , and $1600(=450 \times 3+50 \times 5)$ per modality, respectively.

We constructed the indexes described in Sect. 4.3.1 using $P(N-P)(=32 \times 418)$ scores between templates per modality. We then trained the regression coefficients $\left(w_{1}^{m}, w_{0}^{m}\right)$ and $\left(\tilde{w}_{1}^{m}, \tilde{w}_{0}^{m}\right)$ using the BRLR (Bias-Reduced Logistic Regression) package for $\mathrm{R}$ [35]. We computed one score matrix and one pseudo-score matrix using 450 training 
samples and 450 templates $(450 \times 450$ scores and $418 \times 418$ pseudo-scores between training samples and templates), and used them for training. We also estimated $\operatorname{EER}(N)$ for each modality, which was used to select the non-pivot (as described in Sect. 4.2.1), using the above score matrix.

Then, we evaluated the performance when all the enrollees and non-enrollees input all the fingers 3 times and 5 times, respectively $(450 \times 3 \times 10=13500$ genuine attempts and $50 \times 5 \times 10=2500$ impostor attempts).

\subsubsection{NIST BSSR1 Set3}

The second dataset is the NIST BSSR1 Set3 [18] which contains face scores from 3000 subjects (each subject contributed two samples other than the template) using two algorithms ("C" and "G") $(M=2)$. We excluded 8 subjects whose have inappropriate scores (the values " -1 ") in the algorithm "C". $(2992 \times 2992 \times 2 \times 2$ scores in total; there were no scores between templates). In $\mathbf{P I} \mathbf{I}_{\Phi}-\mathbf{N B F}$, we normalized scores of each algorithm so that the minimum and maximum value would be 0 and 1 , respectively, to keep compatibility of pseudo-scores between two algorithms.

We randomly divided 2992 subjects into 2000 enrollees, 500 non-enrollees, and 492 training subjects (we randomly chose 10 ways to divide), and randomly selected 32 pivots per modality $(N=2000, P=32)$. Then, we divided two samples of each enrollee into the one for constructing the index and the testing sample. As for nonenrollees, we used both the two samples as testing samples. The number of samples for constructing the indexes, training samples, and testing samples was 2000, $984(=492 \times 2)$, and $3000(=2000+500 \times 2)$, respectively.

We constructed the indexes using $P(N-P)(=64 \times$ 1936) scores per matcher, obtained from the above 2000 samples. We then trained the regression coefficients $\left(w_{1}^{m}, w_{0}^{m}\right)$ and $\left(\tilde{w}_{1}^{m}, \tilde{w}_{0}^{m}\right)$ using two score matrices $(2 \times 492 \times$ 492 scores from the training samples) and one pseudo-score matrix (492 $\times 492$ pseudo-scores between the first training samples and the second training samples), obtained from 984 training samples. We also estimated $\operatorname{EER}(N)$ using the above score matrices.

Then, we evaluated the performance when all the enrollees and non-enrollees present their face once and twice, respectively $(2000 \times 1 \times 10=20000$ genuine attempts and $500 \times 2 \times 10=10000$ impostor attempts).

Table 2 shows how we used the samples in each dataset.

\subsection{Experimental Results}

\subsubsection{Indexing and Search Performance}

We first evaluated the trade-off between the penetration rate and the retrieval error rate in $\mathbf{P} \mathbf{I}_{\Phi^{-}}-\mathbf{N B F}, \mathbf{P} \mathbf{I}_{\Pi^{-}} \mathbf{N B F}, \mathbf{P I}_{\Phi^{-}}$ NBS-NBF and $\mathbf{P I}_{\Pi}-\mathbf{N B S}-\mathbf{N B F}$, to investigate the indexing and search performance. Here, we did not use the technique for reducing the average number of score computations (described in Sect. 4.4) in our proposals (PI $\mathbf{I}_{\Phi}$-NBS-NBF and
Table 2 Uses of biometric samples in each dataset

(a) CASIA-FingerprintV5 $(N=450, P=32, M=4)$

\begin{tabular}{l|c|c}
\hline \hline & 450 enrollees & 50 non-enrollees \\
\hline 1st sample (template) & indexing & testing \\
2nd sample & training & testing \\
3rd sample & testing & testing \\
4th sample & testing & testing \\
5th sample & testing & testing \\
\hline
\end{tabular}

(b) NIST BSSR1 Set3 $(N=2000, P=32, M=2)$

\begin{tabular}{l|c|c|c}
\hline \hline & 2000 enrollees & 500 non-enrollees & $\begin{array}{c}492 \text { training } \\
\text { subjects }\end{array}$ \\
\hline $\begin{array}{l}\text { 1st sample } \\
\text { 2nd sample }\end{array}$ & $\begin{array}{c}\text { indexing } \\
\text { testing }\end{array}$ & $\begin{array}{c}\text { testing } \\
\text { testing }\end{array}$ & $\begin{array}{c}\text { training } \\
\text { training }\end{array}$ \\
\hline
\end{tabular}

(a) CASIA-FingerprintV5

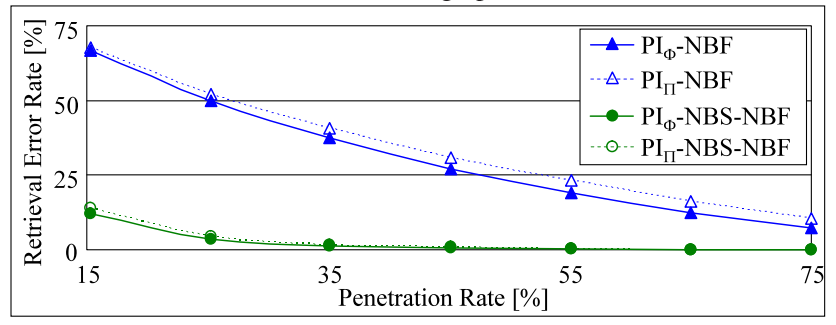

(b) NIST BSSR1 Set3

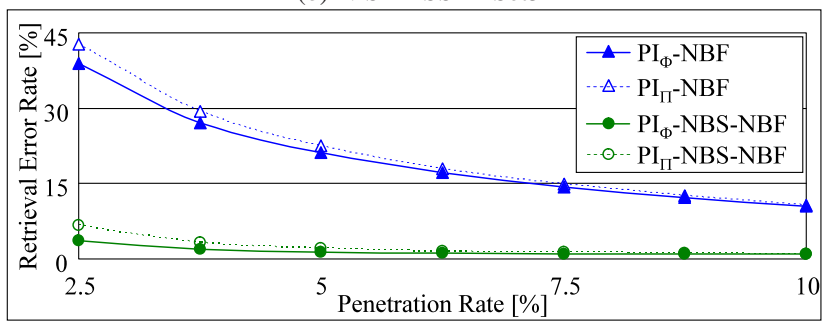

Fig.5 Relationships between the penetration rate and the retrieval error rate in $\mathbf{P I}_{\Phi}$-NBF, $\mathbf{P I}_{\Pi}-\mathbf{N B F}, \mathbf{P I}_{\Phi}$-NBS-NBF, and $\mathbf{P} \mathbf{I}_{\Pi}-\mathbf{N B S}-\mathbf{N B F}$., obtained by changing $T$ (we did not use the technique for reducing the average number of score computations described in Sect. 4.4).

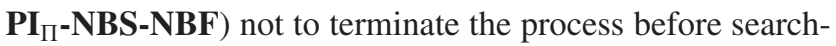
ing all four genuine templates. Then we obtained the tradeoff curves by changing $T$.

Figure 5 shows the results. It was found that $\mathbf{P I}_{\Phi}$-NBSNBF and $\mathbf{P I}_{\Pi}-\mathbf{N B S}-\mathbf{N B F}$ outperformed $\mathbf{P I} \mathbf{I}_{\Phi^{-}} \mathbf{N B F}$ and $\mathbf{P I}_{\Pi^{-}}$ NBF, respectively, by a wide margin, and performed much better in the NIST BSSR1 Set3. For example, when we fixed the retrieval error rate to be $1[\%]$, the penetration rate of $\mathbf{P I}_{\Phi}$-NBS-NBF was 40[\%] in the CASIA-FingerprintV5, while it was 7.5[\%] in the NIST BSSR1 Set3. We explain the reason for this in detail in Sect. 5.2.2.

It was also found that the standard-pivot based indexing scheme $\mathbf{P} \mathbf{I}_{\Phi}$ slightly outperformed the permutation-based indexing scheme $\mathbf{P I}_{\Pi}$. Although Chávez et al. [7] reported that $\mathbf{P} \mathbf{I}_{\Pi}$ outperformed $\mathbf{P} \mathbf{I}_{\Phi}$ in some cases by a wide margin, it was not the case here. We think the reason for this is that the number of pivots was relatively small in our experiments $(P=32)$ and permutations did not have discriminative power, compared to score vectors. In [36], $\mathbf{P I}_{\Phi}$ also outperformed $\mathbf{P I}_{\Pi}$ when $P=16$, while it was not the case 
(a) CASIA-FingerprintV5

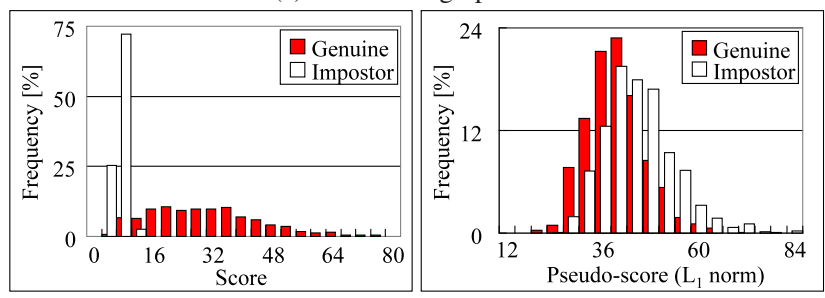

(b) NIST BSSR1 Set3

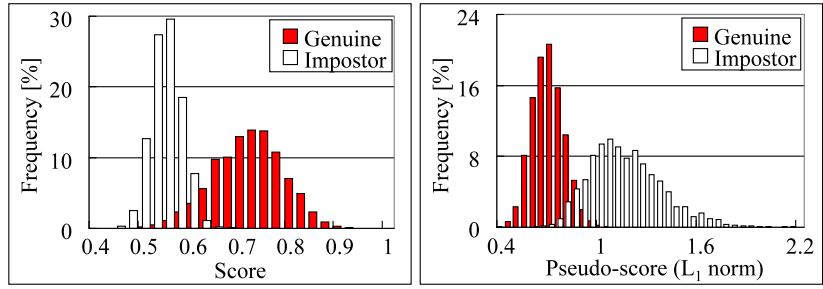

Fig. 6 Frequency distributions of genuine scores (left), impostor scores (left), genuine pseudo-scores (right), and impostor pseudo-scores (right) of the left index fingers, where $\mathbf{P I}_{\Phi}$ is used as an indexing scheme.

when $P=64$.

Thus, in the following, we only report the case where $\mathbf{P I}_{\Phi}$ was used as an indexing scheme (we also confirmed that $\mathbf{P I}_{\Phi}$ provided slightly better identification performance than $\left.\mathbf{P} \mathbf{I}_{\Pi}\right)$.

\subsubsection{Score Distributions and Pseudo-Score Distributions}

In Sect. 5.2.1, $\mathbf{P I}_{\Phi}$-NBS-NBF outperformed $\mathbf{P I}_{\Phi}-\mathbf{N B F}$ by a wide margin, and performed much better in the NIST BSSR1 Set3. To explain the reason for this, we investigated (1) how well scores and pseudo-scores captured discriminative information, and (2) whether the logistic regression model was valid, in both the datasets.

Figure 6 shows the frequency distributions of genuine scores, impostor scores, genuine pseudo-scores, and impostor pseudo-scores of the left index fingers, obtained using 418 non-pivots and the corresponding $1254(=418 \times 3)$ testing samples (although there were 10 ways to divide 500 subjects, we adopted the first one in this analysis). It was found that pseudo-scores captured much less discriminative information than scores, in the CASIA-FingerprintV5. In the CASIA-FingerprintV5, the subjects were asked to rotate their fingers with various levels of pressure to generate significant intra-class variations [17]. Indeed, we can see from Fig. 6 that the variance of genuine scores is much larger than that of impostor scores. Thus, the most likely explanation is that the significant intra-class variations in fingerprint images increased the intra-class variations in score vectors as well, which caused the increase of genuine pseudo-scores and the loss of discriminative power of pseudo-scores.

Figure 7 shows the logarithm of the ratio between the two frequency distributions in Fig. 6, along with the linear functions in the logistic regression model estimated using the training samples. It was found that the logistic regression model was a good fit for modeling the log-likelihood (a) CASIA-FingerprintV5

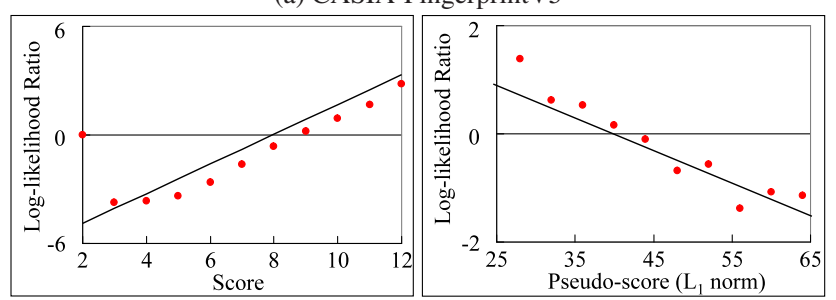

(b) NIST BSSR1 Set3

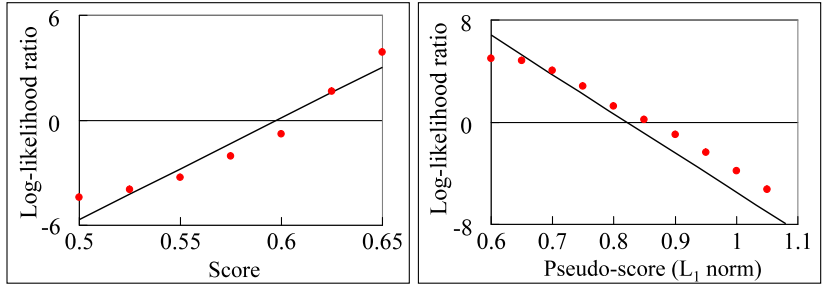

Fig. 7 Logarithm of the ratio between the two frequency distributions in Fig. 6 (left: score, right: pseudo-score). The black lines represent the linear function in logistic regression estimated using the training samples.

ratio functions of scores and pseudo-scores.

Thus, we think the reason that $\mathbf{P I}_{\Phi}$-NBS-NBF outperformed $\mathbf{P I} \mathbf{I}_{\Phi}-\mathbf{N B F}$ was because $\mathbf{P} \mathbf{I}_{\Phi}$-NBS-NBF (1) used not only pseudo-scores but scores, which captured discriminative information, as a clue to find genuine templates, and (2) correctly estimated the log-likelihood ratio functions of scores and pseudo-scores using the logistic regression model. We also think the reason for providing much better performance in the NIST BSSR1 Set3 is that there was much less intra-class variations in face images.

\subsubsection{Identification Performance}

We then evaluated the $\operatorname{FRR}(N)-\operatorname{FAR}(N)$ curves in UB, $\mathbf{P I} \mathbf{I}_{\Phi}-\mathbf{N B F}$ and $\mathbf{P I}_{\Phi}-\mathbf{N B S}-\mathbf{N B F}$. Here, we used the technique for reducing the average number of score computations (described in Sect. 4.4) in $\mathbf{P I}_{\Phi}$-NBS-NBF to identify the claimant on the way of searching non-pivots. Then we set $P R_{r e q}=35[\%]$ (i.e. $T=502$ ) in the CASIA-FingerprintV5, and $P R_{\text {req }}=2.5[\%]$ (i.e. $T=36$ ) in the NIST BSSR1 Set3. We also tried the case where $P R_{r e q}=100[\%]$ in $\mathbf{P} \mathbf{I}_{\Phi}$-NBSNBF, in both the datasets. Then, we obtained the FRR $(N)$ $\operatorname{FAR}(N)$ curves by changing the identification threshold $A$.

Figure 8 shows the results, where the number of scores in parenthesis represents the average number of score computations. It was found that $\mathbf{P I}_{\Phi}-\mathbf{N B S}-\mathbf{N B F}$ significantly improved the accuracy of $\mathbf{U B}$ and $\mathbf{P I}_{\Phi}$-NBF. In the NIST BSSR1 Set3, PI $_{\Phi}$-NBS-NBF also significantly reduced the number of score computations by setting $P R_{\text {req }}=2.5[\%]$. The number of score computations was on average 79 scores and at most 100 scores (i.e. twentieth of that in UB). On the other hand, in the CASIA-FingerprintV5, we confirmed that the accuracy of $\mathbf{P I}_{\Phi}$-NBS-NBF was severely degraded when we reduced $P R_{\text {req }}$ from 35[\%]. The reason for this is that the retrieval error rate was significantly increased, as shown in Fig. 5. 
(a) CASIA-FingerprintV5

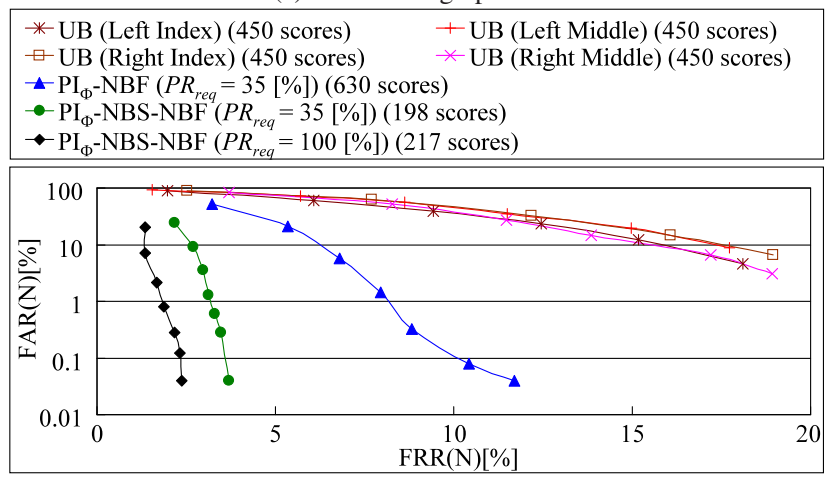

(b) NIST BSSR1 Set3

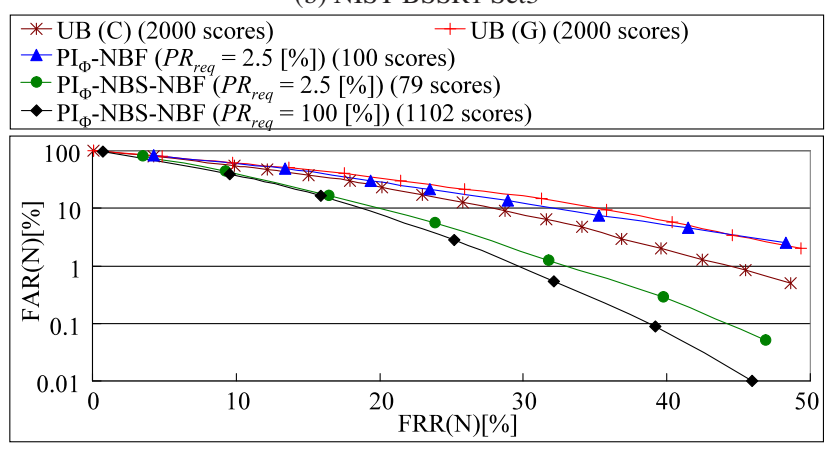

Fig. $8 \operatorname{FRR}(N)$ - $\operatorname{FAR}(N)$ curves in $\mathbf{U B}, \mathbf{P I}_{\Phi}$-NBF and $\mathbf{P I}_{\Phi}$-NBS-NBF, obtained by changing the identification threshold $A$ (we used the technique for reducing the average number of score computations described in Sect. 4.4). The number of scores in parenthesis is the average number of score computations in each method. (a) CASIA-FingerprintV5

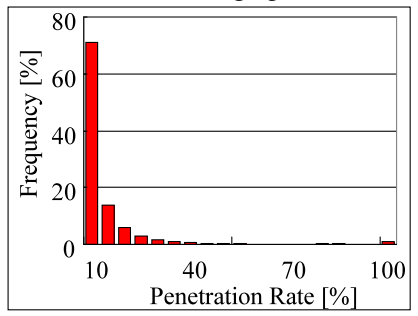

(b) NIST BSSR1 Set3

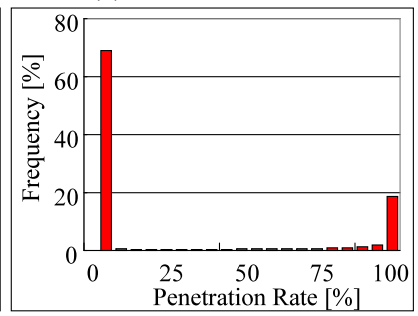

Fig. 9 Frequency distributions of the penetration rate in $\mathbf{P I}_{\Phi}$-NBS-NBF $\left(P R_{r e q}=100[\%]\right)$, in the case where $\operatorname{FAR}(N)=\operatorname{FRR}(N)(=\operatorname{EER}(N))$.

It was $\mathbf{P I}_{\Phi}$-NBS-NBF $\left(P R_{r e q}=100[\%]\right)$ that achieved the best accuracy. We also confirmed that the accuracy of this method was almost the same as that of the NBF scheme which computed all scores [11]. That is, our proposal reduced the average number of score computations without affecting the accuracy, by identifying the claimant on the way of searching non-pivots, as described in Sect. 4.1. The average number of score computations in this method was 217 in the CASIA-FingerprintV5, and 1102 in the NIST BSSR1 Set3, respectively (i.e. about half of that in $\mathbf{U B}$ ).

However, since they were just the average values, we also investigated the detail. Figure 9 shows the frequency distributions of the penetration rate in this method, in the case where $\operatorname{FAR}(N)=\operatorname{FRR}(N)(=\operatorname{EER}(N))$. It was found
Table 3 Average, standard deviation, and coefficient of variation of the retrieval error rate [\%] in the case where we tried 10 ways of randomly selecting 32 pivots (AVG: average, STD: standard deviation, CV: coefficient of variation (= STD/AVG)).

\begin{tabular}{l|c||c|c}
\hline \hline \multicolumn{2}{c|}{} & $\begin{array}{c}\text { CASIA-FingerprintV5 } \\
\left(P R_{\text {req }}=35[\%]\right)\end{array}$ & $\begin{array}{c}\text { NIST BSSR1 Set3 } \\
\left(P R_{\text {req }}=2.5[\%]\right)\end{array}$ \\
\hline \multirow{3}{*}{$\mathbf{P I}_{\Phi}$-NBF } & AVG & 37.3 & 40.6 \\
\cline { 2 - 4 } & STD & 0.191 & 1.76 \\
\cline { 2 - 4 } & CV & 0.00514 & 0.0434 \\
\hline $\mathbf{P I}_{\Pi-N B F}$ & AVG & 40.3 & 44.9 \\
\cline { 2 - 4 } & STD & 0.200 & 2.29 \\
\cline { 2 - 4 } & CV & 0.00495 & 0.0509 \\
\hline PI $_{\Phi}$-NBS-NBF & AVG & 1.28 & 4.15 \\
\cline { 2 - 4 } & STD & 0.0732 & 0.590 \\
\cline { 2 - 4 } & CV & 0.0570 & 0.142 \\
\hline PI $_{\Pi-N B S-N B F}$ & AVG & 1.79 & 8.18 \\
\cline { 2 - 4 } & STD & 0.0661 & 1.35 \\
\cline { 2 - 4 } & CV & 0.0370 & 0.165 \\
\hline
\end{tabular}

that all scores were computed at a rate of $19[\%]$ in the NIST BSSR1 Set3. The reason for this is that the identification error rate was high in this dataset (e.g. $\operatorname{EER}(N)$ of this method was $16[\%])$. That is, in a number of genuine attempts, the system could not find the genuine templates close to the query samples, and had to compute all scores.

\subsubsection{Response Time}

In the CASIA-FingerprintV5, we also measured the time to identify the claimant after obtaining a fingerprint image, on an Intel Core2 Duo CPU E7500 (2.93 GHz) with 1.93GB RAM (we did not measure the time in the NIST BSSR1 Set3 because this was a score dataset). It took on average $1.12[\mathrm{~ms}]$ to compute one score, $73.4[\mathrm{~ms}]$ to extract a feature from a fingerprint image, and the other processing time was negligible. Consequently, the one-to-many matching time can be estimated to be $0.50[\mathrm{~s}]$ in $\mathbf{U B}$, on average $0.22[\mathrm{~s}]$ in $\mathbf{P I}_{\Phi}$-NBS-NBF $\left(P R_{r e q}=35[\%]\right)$, and on average 0.24[s] in $\mathbf{P I}_{\Phi}$-NBS-NBF $\left(P R_{r e q}=100[\%]\right)$, respectively. They can increase in proportion to the number of enrollees $N$.

\subsubsection{Effect of Pivots}

In earlier subsections, we evaluated the performance using randomly selected 32 pivots, as described in Sect. 5.1.1. However, we can expect that the way of randomly selecting pivots can affect the performance. To investigate this effect, we tried 10 ways of randomly selecting 32 pivots, and evaluated the retrieval error rate in the same way as Sect. 5.2.1. Here, we evaluated not the identification performance but the retrieval error rate because it is the retrieval error rate that can be directly affected by the selection of pivots (i.e. we would like to more directly analyze the effect of pivots).

Table 3 shows the average, standard deviation, and coefficient of variations (= standard deviation/average) of the retrieval error rate in the four methods. Here, the penetration rate was fixed to be $P R_{\text {req }}=35[\%]$ (i.e. $T=502$ ) in CASIA-FingerprintV5, and $P R_{r e q}=2.5[\%]$ (i.e. $T=36$ ) in the NIST-BSSR1 Set3. It was found that the coefficients of 
variations in the NIST-BSSR1 Set3 were much larger than those in the CASIA-FingerprintV5, which indicates that the selection of pivots affected the performance especially in the NIST-BSSR1 Set3. We also confirmed that this was true irrespective of the penetration rate $P R_{\text {req }}$ (we changed $P R_{\text {req }}$ to various values, and confirmed that the coefficients of variations in the NIST-BSSR1 Set3 were much larger as well). We consider this may be because pseudo-scores in the NISTBSSR1 Set3 captured much more discriminative information than those in the CASIA-FingerprintV5, as shown in Fig. 6. That is, the effect of pivots may be especially large in the case where pseudo-score based indexing schemes are very effective. Although Chávez et al. [7] showed that the random selection method performed well in their experiments, the best way of selecting pivots is still an open issue. We also leave it as future work.

\subsubsection{Summary and Discussion}

The experimental results can be summed up as follows:

- $\mathbf{P I}_{\Phi}$-NBS-NBF significantly improved the accuracy of UB, while reducing the average number of score computations, in both the datasets.

- $\mathbf{P I}_{\Phi}$-NBS-NBF significantly outperformed $\mathbf{P I}_{\Phi}$-NBF because it used not only pseudo-scores but scores, and the logistic regression model was valid.

- Although $\mathbf{P I}_{\Phi}$-NBS-NBF $\left(P R_{\text {req }}=100[\%]\right)$ reduced the average number of score computations while providing the accuracy almost the same as that of the NBF scheme which computed all scores [11], it had to compute all scores in the worst case.

- $\mathbf{P I}_{\Phi}$ slightly outperformed $\mathbf{P} \mathbf{I}_{\Pi}$ probably due to the small number of pivots $(P=32)$.

- Pseudo-scores had small discriminative power in the CASIA-FingerprintV5 which had large intra-class variations.

- Although we randomly selected pivots, the way of randomly selecting pivots affected the performance.

Although Lemma 1 in Sect. 4.2.2 only states the optimality of the NBS scheme in a limited sense, we believe that this lemma also contributed as a theoretical basis for why our proposal performed very well in our experiments.

Our proposal reduced the average number of score computations less than twentieth of that in UB, in the NIST BSSR1 Set3. On the other hand, it only reduced to about half in the CASIA-FingerprintV5. However, it still provides great value to the large-scale identification system thanks to general applicability as described in Sect. 2.2: it can be applied to any kind of modality and even to commercial systems which do not provide access to the features. We think the response time would be further reduced by combining our proposal and feature level classification (or indexing) [19]-[24], which is left as future work.

\section{Conclusion}

In this paper, we first proposed a general framework for score level indexing and fusion, and then we proposed the NBS scheme and discussed its optimality with regard to the retrieval error rate. The experimental results showed that our proposal significantly improved the accuracy of the unimodal biometrics, while reducing the average number of score computations. However, except in the case of multiple matchers, we need to keep in mind that our solution may cause inconvenience because the claimant has to input multiple different biometrics. We are now planning to extend our framework to the sequential fusion scenario [12], where the system identifies the claimant each time he/she inputs a query sample, to keep down the number of query samples the claimant has to input.

Other future work includes the following: 1) estimation of the log-likelihood ratio which takes into the account the correlation between scores and pseudo-scores; 2) automatic determination of the optimal number of pivots and selecting the best pivots with regard to the retrieval error rate; 3) combination of our proposal and feature level classification (or indexing).

\section{References}

[1] T. Murakami and K. Takahashi, "Fast and accurate biometric identification using score level indexing and fusion," Proc. International Joint Conference on Biometrics (IJCB '11), 2011.

[2] R.M. Bolle, J.H. Connell, S. Pankanti, N.K. Ratha, and A.W. Senior, Guide to Biometrics, Springer, 2003.

[3] M. Tistarelli, S.Z. Li, and R. Chellappa, Handbook of Remote Biometrics: For Surveillance and Security, Springer, 2009.

[4] A. Ross and A.K. Jain, "Multimodal biometrics: An overview," Proceedings of the 12th European Signal Pocessing Conference (EUSIPCO), pp.1221-1224, 2004.

[5] E. Chávez, G. Navarro, R. Baeza-Yates, and J.L. Marroquín, "Searching in metric spaces," ACM Computing Surveys, vol.33, no.3, pp.273-321, 2001.

[6] M. Patella and P. Ciaccia, "Approximate similarity search: A multi-faceted problem," Journal of Discrete Algorithms, vol.7, no.1, pp.36-48, 2009.

[7] E. Chávez, K. Figueroa, and G. Navarro, "Effective proximity retrieval by ordering permutations," IEEE Trans. Pattern Anal. Mach. Intell., vol.30, no.9, pp.1647-1658, 2008.

[8] G. Amato and P. Savino, "Approximate similarity search in metric spaces using inverted files," Proceedings of the 3rd international conference on Scalable information systems (InfoScale '08), pp.1$10,2008$.

[9] O. Edsberg and M.L. Hetland, "Indexing inexact proximity search with distance regression in pivot space," Proceedings of the Third International Workshop on Similarity Search and Applications (SISAP '10), pp.51-58, 2010.

[10] V. Athitsos, J. Alon, S. Sclaroff, and G. Kollios, "Boostmap: An embedding method for efficient nearest neighbor retrieval," IEEE Trans. Pattern Anal. Mach. Intell., vol.30, no.1, pp.89-104, 2008.

[11] K. Nandakumar, A.K. Jain, and A. Ross, "Fusion in multibiometric identification systems: What about the missing data?," Proceedings of the Third International Conference on Advances in Biometrics (ICB '09), pp.743-752, 2009.

[12] T. Murakami and K. Takahashi, "Accuracy improvement with high 
convenience in biometric identification using multihypothesis sequential probability ratio test," Proc. First IEEE International Workshop on Information Forensics and Security (WIFS '09), pp.66-70, 2009.

[13] K. Nandakumar, Y. Chen, S.C. Dass, and A.K. Jain, "Likelihood ratio-based biometric score fusion,' IEEE Trans. Pattern Anal. Mach. Intell., vol.30, no.2, pp.342-347, 2008.

[14] O. Fatukasi, J. Kittler, and N. Poh, "Estimation of missing values in multimodal biometric fusion," Proc. 2nd IEEE International Conference on Biometrics: Theory, Applications and Systems (BTAS '08), 2008.

[15] N. Poh, D. Windridge, V. Mottl, A. Tatarchuk, and A. Eliseyev, "Addressing missing values in kernel-based multimodal biometric fusion using neutral point substitution," IEEE Trans. Information Forensics and Security, vol.5, no.3, pp.461-469, 2010.

[16] Y. Ding and A. Ross, "When data goes missing: Methods for missing score imputation in biometric fusion," Proc. SPIE Conference on Biometric Technology for Human Identification VII, 2010.

[17] CASIA-FingerprintV5. http://biometrics.idealtest.org/

[18] NIST Biometric Scores Set - Release 1 (BSSR1). http://www.nist. gov/itl/iad/ig/biometricscores.cfm

[19] D. Maltoni, D. Maio, A.K. Jain, and S. Prabhakar, "Fingerprint classification and indexing," in Handbook of Fingerprint Recognition, second ed., ch. 5, pp.235-269, Springer, 2009.

[20] O. Iloanusi, A. Gyaourova, and A. Ross, "Indexing fingerprints using minutiae quadruplets," Proc. of 2011 Conference on Computer Vision and Pattern Recognition Workshops (CVPRW'11), pp.127$133,2011$.

[21] X. Liang, A. Bishunu, and T. Asano, "A robust fingerprint indexing scheme using minutia neighborhood structure and low-order delaunay triangles," IEEE Trans. Information Forensics and Security, vol.2, no.4, pp.721-733, 2007.

[22] X. Shuai, C. Zhang, and P. Hao, "Fingerprint indexing based on composite set of reduced sift features," Proc. 19th International Conference on Pattern Recognition (ICPR '08), 2008.

[23] M.H. Izadi, L. Mirmohamadsadeghi, and A. Drygajlo, "Introduction of cylinder quality measure into minutia cylinder-code based fingerprint matching," Proc. of the Fifth International Conference on Biometrics: Theory, Applications and Systems (BTAS'12), pp.353358, 2012.

[24] R. Cappelli, M. Ferrara, and D. Maltoni, "Fingerprint indexing based on minutia cylinder-code," IEEE Trans. Pattern Anal. Mach. Intell., vol.33, no.5, pp.1051-1057, 2011.

[25] R.O. Duda, P.E. Hart, and D.G. Stork, Pattern Classification, WileyInterscience, 2000.

[26] G. Becker and M. Potts, "Non-metric biometric clustering," Proceedings of Biometrics Symposium, pp.1-6, 2007.

[27] T. Maeda, M. Matsushita, and K. Sasakawa, "Identification algorithm using a matching score matrix," IEICE Trans. Inf. \& Syst., vol.E84-D, no.7, pp.819-824, July 2001.

[28] A. Gyaourova and A. Ross, "Index codes for multibiometric pattern retrieval," IEEE Trans. on Information Forensics and Security, vol.7, no.2, pp.518-529, 2012.

[29] J. Daugman, "Probing the uniqueness and randomness of iriscodes: Results from 200 bilion iris pair comparisons," Proc. IEEE, vol.94, no.11, pp.1927-1935, 2006.

[30] L. Micó, J. Oncina, and E. Vidal, "An algorithm for finding nearest neighbours in constant average time with a linear space complexity," Proc. 11th International Conference on Pattern Recognition (ICPR 1992), pp.557-560, 1992.

[31] C.M. Bishop, Pattern Recognition and Machine Learning, Springer, 2006.

[32] P. Verlinde and M. Acheroy, "A contribution to multi-modal identity verification using decision fusion," Proc. PROMOPTICA, 2000.

[33] K. Nandakumar, A. Ross, and A.K. Jain, "Biometric fusion: Does modeling correlation really matter?," Proc. IEEE 3rd International Conference on Biometrics: Theory, Applications, and Systems
(BTAS '09), pp.271-276, 2009.

[34] SourceAFIS. http://sourceforge.net/projects/sourceafis/

[35] P. Wessa, Free Statistics Software, Office for Research Development and Education, version 1.1.23-r7, 2012. http://www.wessa.net/

[36] T. Murakami, K. Takahashi, S. Serita, and Y. Fujii, "Probabilistic enhancement of approximate indexing in metric spaces," Information Systems Journal, Elsevier, vol.38, no.7, pp.1007-1018, 2013.

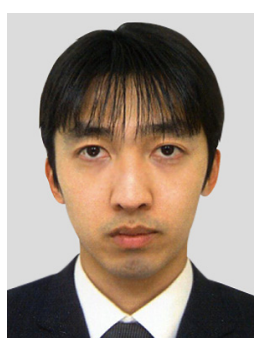

Takao Murakami is a researcher of the Yokohama Laboratory, Hitachi, Ltd. He received the BS degree and the MS degree from the University of Tokyo in 2004 and 2006. He joined Hitachi, Ltd. in 2006. Since then, he has worked on research and development of biometric authentication systems. He is now a doctoral candidate in Institute of Industrial Science, the University of Tokyo. He received the Yamashita SIG Research Award from the Information Promember of IEICE, IPSJ and IEEE. cessing Society of Japan (IPSJ) in 2011. He is a

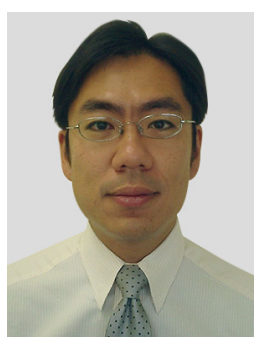

Kenta Takahashi is a researcher of the Yokohama Laboratory, Hitachi, Ltd. He received the BS degree and the MS degree from the University of Tokyo in 1998 and 2000. He joined Hitachi, Ltd. in 2000. Since then, he has worked on research and development of biometric authentication systems. He is now a doctoral candidate in the Graduate School of Information Science and Technology, The University of Tokyo. He received the best paper award from the Information Processing Society

of Japan (IPSJ) in 2009. He is a member of IEICE, IPSJ and IEEE.

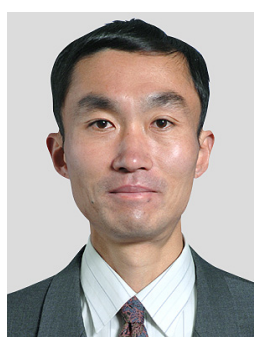

Kanta Matsuura received B.Eng., M.Eng., and $\mathrm{Ph} . \mathrm{D}$. degrees from the University of Tokyo, Japan, in 1992, 1994, and 1997, respectively. $\mathrm{He}$ is currently an associate professor at Institute of Industrial Science, the University of Tokyo. His research interests include cryptography, network security, and security management. In 2008, he won Distinguished-Service Award from the IEICE Communications Society. He served as an associate editor of IEICE Transactions on Communications between 2005 and 2008. He has been serving as the editor-in-chief of JSSM (Japan Society of Security Management) Journal since 2008, and a member of Editorial Board of Designs, Codes and Cryptography since 2010. He was a program co-chair of IWSEC2008, and a general co-chair of IWSEC2011. He is a member of IACR and IPSJ. He is a senior member of ACM, IEEE, and IEICE. He is a member of the Board-of-Directors of JSSM. 